OPEN ACCESS

Edited by:

Xihui Shen,

Northwest A\&F University, China

Reviewed by:

Joseph James Gillespie,

University of Maryland, Baltimore,

United States

Shitao Li,

Oklahoma State University,

United States

*Correspondence:

Jere W. McBride

jemcbrid@utmb.edu

Received: 02 September 2017 Accepted: 22 December 2017 Published: 11 January 2018

Citation:

Farris TR, Zhu B, Wang JY and McBride JW (2018) Ehrlichia chaffeensis TRP32 Nucleomodulin Function and Localization Is Regulated by NEDD4L-Mediated Ubiquitination.

Front. Cell. Infect. Microbiol. 7:534 doi: $10.3389 / f c i m b .2017 .00534$

\section{Ehrlichia chaffeensis TRP32 Nucleomodulin Function and Localization Is Regulated by NEDD4L-Mediated Ubiquitination}

\author{
Tierra R. Farris ${ }^{1}$, Bing Zhu ${ }^{2}$, Jennifer Y. Wang ${ }^{3}$ and Jere W. McBride ${ }^{1,2,3,4,5,6 *}$ \\ ${ }^{1}$ Departments of Microbiology and Immunology, University of Texas Medical Branch, Galveston, TX, United States, \\ ${ }^{2}$ Pathology, University of Texas Medical Branch, Galveston, TX, United States, ${ }^{3}$ Cell Biology, University of Texas Medical \\ Branch, Galveston, TX, United States, ${ }^{4}$ Center for Biodefense and Emerging Infectious Diseases, University of Texas Medical \\ Branch, Galveston, TX, United States, 5 Sealy Center for Vaccine Development, University of Texas Medical Branch, \\ Galveston, TX, United States, ${ }^{6}$ Institute for Human Infections and Immunity, University of Texas Medical Branch, Galveston, \\ TX, United States
}

Ehrlichia chaffeensis is an obligately intracellular bacterium that reprograms the mononuclear phagocyte through diverse effector-host interactions to modulate various host cell processes. In a previous study, we reported that the E. chaffeensis nucleomodulin TRP32 regulates transcription of host genes in several biologically relevant categories, including cell differentiation and proliferation. In this study, we investigate the effect of ubiquitination on TRP32 function and localization within the host cell. TRP32 is both mono- and polyubiquitinated on multiple lysine residues during infection and when ectopically expressed. Despite lacking a canonical PPXY motif, TRP32 interacted with, and was modified by the human HECT E3 ubiquitin (Ub) ligase NEDD4L. TRP32 ubiquitination was not by K48-linked polyUb chains, nor was it degraded by the proteasome; however, TRP32 was modified by K63-linked polyUb chains detected both in the cytosol and nucleus. HECT ligase inhibitor, heclin, altered the subnuclear localization of ectopically expressed TRP32 from a diffuse nuclear pattern to a lacy, punctate pattern with TRP32 distributed around the periphery of the nucleus and nucleoli. When a TRP32 lysine null (K-null) mutant was ectopically expressed, it exhibited a similar phenotype as single lysine mutants (K63R, K93R, and K123R). However, the K-null mutant showed increased amounts of cytoplasmic TRP32 compared to single lysine mutants or heclin-treated cells ectopically expressing TRP32. These alterations in localization corresponded to changes in TRP32 transcriptional repressor function with heclin-treated and single lysine mutants unable to repress transcription of a TRP32 target genes in a luciferase assay.

Keywords: Ehrlichia, ubiquitination, post-translational modification, effector, NEDD4L, localization, tandem repeat protein

\section{INTRODUCTION}

Ehrlichia chaffeensis is a gram-negative, obligately intracellular bacterium and the etiologic agent of human monocytotropic ehrlichiosis (HME), an emerging life-threatening tick-borne zoonosis. In humans, E. chaffeensis preferentially infects mononuclear phagocytes, causing an acute infection that manifests as an undifferentiated febrile illness. The mechanisms by which E. chaffeensis 
reprograms various host cell processes is not fully understood; however, a group of type 1 secreted, tandem repeat protein (TRP) effectors similar to the repeats-in-toxin family of exoproteins are involved. TRPs were initially identified as E. chaffeensis major immunoreactive proteins, and are known to elicit protective antibody responses (Kuriakose et al., 2012). Recent studies have revealed that TRPs are secreted pleotropic effectors that interact with a large group of functionally diverse host cell proteins as well as host cell DNA (Lina et al., 2016b).

The most well-characterized TRP effectors, TRP120 and TRP32, interact with many host cell targets, directly activate cell signaling pathways, and activate/repress host cell transcription. Surface-expressed TRPs contribute to ehrlichial entry via WNT pathway activation (Luo et al., 2015). Additionally, TRP120 interactions with ADAM17 on the host cell surface activate the Notch pathway, resulting in the downregulation of innate immune toll-like receptors (Lina et al., 2016a). TRP120 and TRP32 also act as nucleomodulins that manipulate host gene transcription via direct interactions with host target genes. TRP120 binds a GC-rich motif, leading to upregulation of specific host genes involved in transcriptional regulation, signal transduction, and apoptosis (Zhu et al., 2011). TRP32 also binds a G-rich motif consisting of imperfect GGTGGC-like sequence repeats, but preferentially targets genes regulating cell proliferation and differentiation. TRP32 was also shown to activate and repress expression of targets in a gene-specific manner during infection and in a luciferase reporter assay (Luo and McBride, 2012; Farris et al., 2016).

A common theme among bacterial pathogens is the hijacking of host post-translational machinery to modify effectors (Ribet and Cossart, 2010; Ravikumar et al., 2015; Popa et al., 2016). E. chaffeensis effectors are phosphorylated, ubiquitinated, and SUMOylated by host enzymes (McBride et al., 2011; Wakeel et al., 2011; Dunphy et al., 2014; Farris et al., 2016; Zhu et al., 2017), and these PTMS are important for effector function. TRP120 ubiquitination and SUMOylation is required for interactions with host proteins, and TRP32 tyrosine phosphorylation plays a role in its nuclear localization.

Ubiquitination is the covalent attachment of the small peptide modifier ubiquitin (Ub) that occurs via an enzymatic cascade requiring the sequential action of three classes of enzymes the third of which, the E3 Ub ligase ( 600 known), determines substrate specificity (Metzger et al., 2012). Ubiquitination occurs either singly (monoubiquitination) or as a chain covalently linked via any one of seven Ub lysine residues or attached to the N-terminus. All homotypically-linked chains as well as heterotypic and branched polyUb chains have been detected in cells, which direct the substrates to different fates within the cell (Ub and Ub-like proteins as multifunctional signals). The most studied Ub modifications are K48-linked chains which direct proteasomal degradation of target substrates, K63-linked chains which are involved in cell signaling, receptor endocytosis, and protein-protein interactions, and K11-linked chains which may play a role in cell cycle-specific protein degradation (Komander, 2009).

Although bacteria do not possess an endogenous Ub system, the interaction of bacterial effectors with the ubiquitin system is well-described with bacterial proteins acting as both $\mathrm{Ub}$ substrates and ligases (Thomas and Holden, 2009; Collins and Brown, 2010). When bacterial proteins are modified by ubiquitin, these modifications can influence both physical and temporal localization of the protein depending on whether the $\mathrm{Ub}$ conjugate is a single $\mathrm{Ub}$ or a polyUb chain. Monoubiquitination typically alters protein localization and is involved in both nuclear import and export of eukaryotic proteins (Salmena and Pandolfi, 2007). Salmonella is known to exploit ubiquitination to alter subcellular localization of the SopB effector (Patel et al., 2009). SopB is a phosphatase that first localizes to the host membrane, where its enzymatic functions alter actin organization, facilitating bacterial entry. After SopB is multiply monoubiquitinated, it trafficks to the bacteria-containing vacuole where it alters vesicular trafficking to facilitate bacterial replication (Anderson and Frank, 2012). The functional equivalent of polyubiquitination is typically dependent on chain type. However, one of the best characterized examples is the Legionella effector SidH, which utilizes K48linked polyubiquitination for temporal regulation (Ashida et al., 2014).

Although examples of pathogen effectors with PTMassociated functions exist, ehrlichial effector regulation by ubiquitination has previously only been described for TRP120 in which ubiquitination has been linked to protein-protein interactions (Zhu et al., 2017). In this study, we examined the role of ubiquitination on TRP32 transcription factor function. We demonstrate that multiple species of mono- and polyubiquitinated TRP32 can be detected during infection, and that the host E3 enzyme, NEDD4L, ubiquitinates TRP32. Moreover, we found that these $\mathrm{Ub}$ modifications are required for TRP32 transcription factor function and subnuclear localization.

\section{MATERIALS AND METHODS}

\section{Cell Culture and Infection}

Ehrlichia chaffeensis (Arkansas strain) was propagated in a human monocytic cell line (THP-1; ATCC, Manassas, VA). THP-1 cells were maintained in RPMI 1640 with HEPES $(25 \mathrm{mM})$ (Invitrogen; Carlsbad, CA) supplemented with $10 \%$ fetal bovine serum (FBS)(HyClone, Logan, UT), $5 \mathrm{mM} \mathrm{L-}$ glutamine, $1 \%$ sodium pyruvate, and $12.5 \mathrm{ml} 10 \%$ glucose (Sigma; St. Louis, $\mathrm{MO}$ ) at $37^{\circ} \mathrm{C}$ in a $5 \% \mathrm{CO}_{2}$ atmosphere. $E$. chaffeensis infection was maintained by subculturing infected cells with uninfected. HeLa cells (human cervical epithelial; ATCC) for transfection were grown in MEM (Invitrogen) supplemented with $10 \%$ FBS (HyClone) and maintained in a $5 \% \mathrm{CO}_{2}$ atmosphere. When stated cells were incubated with $10 \mathrm{mM}$ bortezomib (ApexBio; Houston, TX), PYR41 (Thermo Fisher; Boston, MA), Heclin (Sigma), carfilzomib (ApexBio) or vehicle (DMSO) for 12-24 h before harvesting cells for lysate or luciferase expression measurement.

\section{Expression and Purification of Recombinant TRP32}

Full-length TRP32 was PCR amplified from E. chaffeensis genomic material and cloned into pGEX-6p1 vectors (GE Healthcare; Piscataway, NJ). The constructs were transformed into BL21 E. coli (Genlantis; San Diego, CA) for protein 
expression. Briefly, overnight cultures were diluted 1:20 in LB plus ampicillin (Amp) and grown for $3 \mathrm{~h}$ with agitation at $37^{\circ} \mathrm{C}$ then protein expression was induced by adding isopropyl- $\beta$ D-thiogalactoside (IPTG) to a final concentration of $0.5 \mathrm{mM}$ and growing for another for $3-4 \mathrm{~h}$ at $37^{\circ} \mathrm{C}$. Cells were then suspended in Tris-buffered saline with protease inhibitors (cOmplete mini, EDTA free) (Sigma), lysed by sonication, then cleared by centrifuging for $20 \mathrm{~min}$ at $12,000 \mathrm{~g}$ at $4^{\circ} \mathrm{C}$. Cleared lysate was then added to washed Glutathione Sepharose $4 \mathrm{~B}$ (GE) and recombinant proteins were purified according to the manufacturer's instructions.

\section{In Vitro Microfluidic Peptide Ubiquitination Array}

Peptides (12-mer) corresponding to TRP32 putative ubiquitination targets were synthesized by flanking a central lysine residue with $6 \mathrm{~N}$-terminal amino acids and $5 \mathrm{C}$-terminal amino acids. For each peptide, a corresponding negative control sequence was included with alanine (A) substituted in place of $\mathrm{K}$ (A peptide). Peptides were synthesized on chip and an in vitro ubiquitination assay performed as previously described (Zhu et al., 2016). Briefly, ubiquitination reactions were performed using an in vitro ubiquination kit (Enzo Life Sciences; Farmingdale, NY) in the presence of THP-1 lysate. Nonspecifically bound proteins were removed by washing with buffer containing $1 \%$ SDS, $0.1 \% \beta$-mercaptoethanol and $100 \mathrm{mM}$ Tris for $30 \mathrm{~min}$, followed by a PBS wash containing 1\% Tween-20 (PBST) then Ub was detected using anti-Ub Alexa Fluor 647 antibody (Santa Cruz Biotechnology, 1:300). The signal was then read using an Anon GenePix 4400A (Molecular devices) scanner running GenePix Pro 7 software. Positive signals were determined by comparison with the control peptides and negative controls for each assay. Differences between wild-type and control peptides were assessed using two-tailed Student's $t$-test. The data are presented as mean $\pm \mathrm{SD}$, and significance was indicated by a $p<0.05$.

\section{In Vitro Ubiquitination Assay}

TRP32 ubiquitination was performed with recombinant TRP32 and an in vitro ubiquitination kit (Enzo Life Science). Briefly, TRP32 (200 nM) was added to ubiquitination buffer, biotintagged $\mathrm{Ub}$ protein, E1 ligase and the E2 ligase $\mathrm{UBCH} 5 \mathrm{~b}$ with and without the E3 NEDD4L and Mg-ATP. Reaction mixtures were incubated at $30^{\circ} \mathrm{C}$ for $3 \mathrm{~h}$ and then boiled with $1 \mathrm{x}$ lithium dodecyl sulfate (LDS) sample buffer. Samples were separated by SDS-PAGE and proteins detected by Western blotting with rabbit anti-TRP32 $(1: 10,000)$ or anti-Ub (FK2, Enzo, 1:1,000) primary antibodies followed by horseradish peroxidase-conjugated antirabbit or anti-mouse secondary antibodies (1:10,000) (KPL; Gaithersburg, MD). Bound antibodies were visualized after incubation with ECL substrate (Pierce; Rockford, IL).

\section{Cotransfection and HA Immunoprecipitation}

HeLa cells were cotransfected with pAcGFP1-C or pAcGFP1TRP32, and WT or lysine null HA-tagged Ub constructs by use of Lipofectamine 2000 (Invitrogen). Cells were collected and lysed in $20 \mathrm{mM}$ Tris, $\mathrm{pH} 7.5,150 \mathrm{mM} \mathrm{NaCl}, 1 \mathrm{mM}$ EDTA, $20 \mathrm{mM}$ NEM (covalent isopeptidase inhibitor; Sigma), 1\% Triton X-100, and protease inhibitors (Complete mini, EDTA-free; Sigma) at $24 \mathrm{~h}$ post-transfection. Lysates were then centrifuged at $4^{\circ} \mathrm{C}$ and $16,000 \mathrm{~g}$ (Eppendorf 5430R centrifuge with model FA 45-30-11 rotor) for $20 \mathrm{~min}$, and the supernatants were incubated with antiHA agarose (Thermo Scientific) overnight at $4^{\circ} \mathrm{C}$. The resin was washed with lysis buffer, boiled in LDS buffer, and analyzed by SDS-PAGE and Western blotting.

\section{Antibody Crosslinking and Immunoprecipitation}

Rabbit anti-TRP32 and control pre-immune serum were crosslinked to Protein A/G Plus Agarose using the Pierce Crosslink IP kit according to manufacturer's instructions (Luo et al., 2008). Briefly, 20 ug of antibody was incubated with washed $A / G$ resin for $1 \mathrm{~h}$ at room temperature. Resin was then washed with PBS to remove unbound antibody. The chemical crosslinker disuccinimidyl suberate (DSS) was added and sample was incubated again for $1 \mathrm{~h}$ at room temperature. Resin was then washed $3 \mathrm{x}$ with elution buffer, then $2 \mathrm{x}$ in wash buffer to remove un-crosslinked antibody. Resin was then stored at $4^{\circ}$ in PBS until use. For immunoprecipitation, lysate was harvested from heavily infected THP-1 cells. Cells were pelleted at $4^{\circ} \mathrm{C}$ and washed $2 \mathrm{x}$ with ice cold PBS. Then cells were resuspended in lysis buffer (TBS with 1\% Triton X-100, cOmplete protease inhibitor (Sigma), $1 \mathrm{mM}$ phenylmethylsulfonylflouride, $1 \mathrm{mM}$ ethylenediaminetetraacetic acid, and $50 \mathrm{mM}$ N-ethylmaleimide) and incubated for $30 \mathrm{~min}$ on ice, vortexing for $30 \mathrm{~s}$ every $5 \mathrm{~min}$. Lysate was then centrifuged at $12,000 \mathrm{~g}$ for $15 \mathrm{~min}$ to clear and soluble fraction retained. Lysate was then pre-cleared by incubating for $30 \mathrm{~min}$ with control agarose resin. Cleared lysate (500 to $1,000 \mu \mathrm{g}$ ) was added to TRP32 and control resin and incubated, on a rotator, at $4^{\circ} \mathrm{C}$ overnight. Resin was then washed 4-6x with lysis buffer and then immunoprecipitated proteins were eluted in $50 \mu$ l elution buffer (Pierce). Ubiquitin enrichment was performed using a Ubiquitin Enrichment kit (Pierce) according to manufacturer's instructions.

\section{Localization}

TRP32 lysine mutants were created from TRP32 in a pACGFP-CI (Clontech; Mountain View, CA) backbone using a QuikChange Mutagensis II kit (Agilent; Santa Clara, CA), or were obtained from a commercial vendor (GenScript). Mutants were amplified in XL1-Blue E. coli and purified using the PerfectPrep EndoFree Plasmid Maxi Kit (5 Prime). Purified plasmids were transfected into HeLa cells using Lipofectamine 2000 (Thermo Fisher) per manufacturer's instructions. Cells were acetone fixed and mounted with ProLong Gold Antifade reagent with DAPI (4',6-diamidino-2-phenylindole) (Invitrogen). Localization of ectopically expressed constructs was examined at $24 \mathrm{~h}$ post-transfection using immunofluorescence microscopy and confocal microscopy. For immunofluorescence microscopy, cells were incubated with mouse anti-B23 (1:100)(Santa Cruz; Santa Cruz, CA) or mouse anti-coilin (1:100)(Santa Cruz) for $1 \mathrm{~h}$, then washed and incubated with Alexa Fluor 594-IgG(H+L) labeled goat anti-mouse secondary antibody (1:100) (Molecular 
Probes; Carlsbad, CA) for $1 \mathrm{~h}$ then mounted with ProLong Gold Antifade reagent with DAPI (Invitrogen). For confocal HeLa cells were seeded unto a glass cover slip, TRP32-GFP expression constructs were transfected (Lipofectamine 2000) and cells harvested $24 \mathrm{~h}$ post-transfection and fixed in $4 \%$ paraformaldehyde in PBS for $20 \mathrm{~min}$ at room temperature. Cells were then permeabilized using $1 \%$ Triton X-100 with $5 \%$ bovine serum albumin (BSA) in PBS for $1 \mathrm{~h}$. After permeabilization, cells were incubated with rabbit anti-TRP32 (1:1000) for $30 \mathrm{~min}$, then washed and labeled with Alexa Fluor 488$\operatorname{IgG}(\mathrm{H}+\mathrm{L})$ conjugated goat anti-rabbit secondary antibodies (1:100; Molecular Probes) for $30 \mathrm{~min}$ before mounting with ProLong Gold antifade reagent with DAPI (4',6-diamidino2-phenylindole) (Invitrogen). Samples were examined using a Zeiss LSM 510 meta laser scanning confocal microscope configured with an Axiovert 200M inverted microscope using a c-Apochromat $63 \mathrm{x} / 1.2$ numerical aperture water immersion lens. UV argon, visible argon ion and green helium neon lasers were used and emissions read using $385-470 \mathrm{~nm}$ (DAPI), 505-530 nm (Alexa Fluor 488-conjugate), 560-615 nm (Alexa Fluor 594-conjugate) band pass filters, respectively. Images were analyzed with LSM 510 software and Z-stacks were constructed by imaging optical slices at $1 \mu \mathrm{M}$ intervals. FIJI was used for subsequent image processing and only linear adjustments (i.e., brightness, contrast) were made (Schindelin et al., 2012).

\section{Luciferase Gene Expression Assay}

Promoter regions containing the TRP32 binding sites as determined using the Genomic Regions Enrichment of Annotations Tool (GREAT) were cloned into the promoterless firefly luciferase vector pGL4.10 (Promega; Madison, WI)(McLean et al., 2010). Each promoter construct (200 ng) was transfected into HeLa cells using Lipofectamine 2,000 (Thermo Fisher) along with GFP-TRP32, or an empty GFP expressing vector and a control vector expressing Renilla luciferase under the control of a HSV-thymidine kinase promoter (pRL-TK Vector, Promega). Equal amounts of total DNA were transfected into each cell. After $24 \mathrm{~h}$, cells were harvested and Dual Glo Luciferase Reagent (Promega) added to the cells according to the manufacturer's protocol. Relative light output was measured using a Veritas Microplate Luminometer. Relative expression levels were calculated for each gene compared to the control using a Student's $t$-test.

\section{RESULTS}

\section{TRP32 Is Mono- and Polyubiquitinated at Multiple Residues}

In silico analysis identified multiple lysine residues in TRP32 that were predicted to be sites of ubiquitination, two of which were proximal to predicted transcriptional transactivation domains (TAD) (Figure 1A; Radivojac et al., 2010). When 12mer peptides corresponding to putative TRP32 ubiquitination sites were examined in vitro using a microfluidic peptide array for their ubiquitination potential, two sites were identified

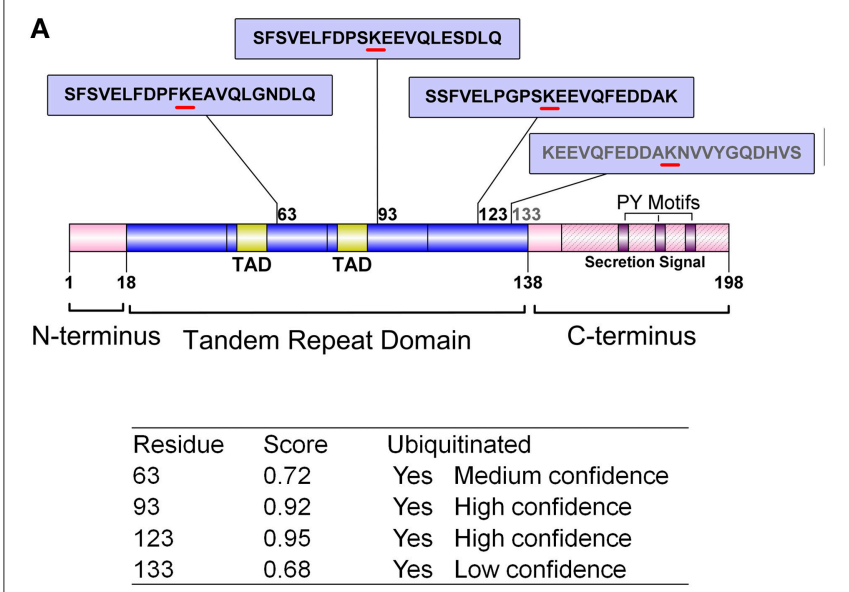

B

TRP32 Ubiquitination Peptide Array

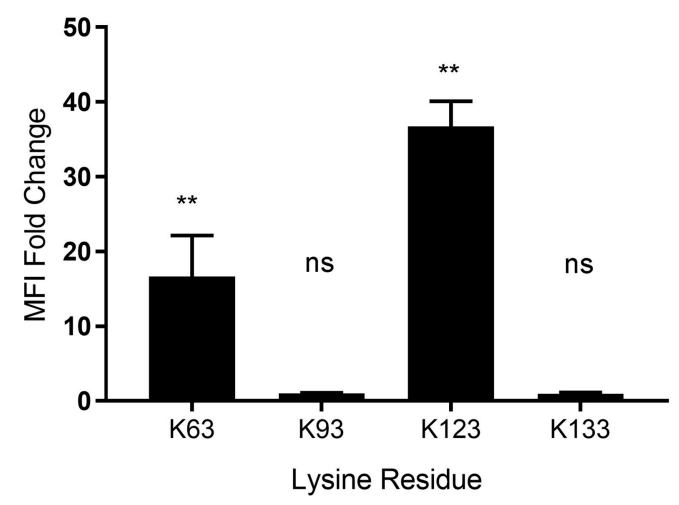

FIGURE 1 | Schematic of $E$. chaffeensis TRP32 domains and predicted sites of ubiquitination and in vitro ubiquitination of TRP32 lysine residues using a peptide array. (A) TRP32 contains four lysine residues, all of which are located within the tandem repeat domain and are predicted to be ubiquitinated with varying confidence levels. Proline-Tyrosine (PY) motifs: TPYY(165-168), NPYY(176-179), TPDY(185-188) are also shown. (B) TRP32 lysine containing peptides were tested in a microfluidic peptide ubiquitination assay. Lysine 63 and 123 showed significantly higher signals than corresponding peptides in which the lysine residues were substituted with alanine.

(Figure 1B). In order to determine if TRP32 was ubiquitinated in human cells, we ectopically expressed GFP-tagged TRP32 along with HA-tagged wildtype (WT) or K-null Ub and performed HA-immunoprecipitation. Unmodified TRP32 migrates at approximately $32 \mathrm{kDa}$, higher than expected due to its low pI (3.44) and hydrophilicity (Luo et al., 2008; Shirai et al., 2008). Multiple high molecular weight TRP32 bands were observed when ectopically expressed TRP32 was co-precipitated with HA-tagged WT or K-null Ub that is unable to form polyUb chains. Fewer high molecular weight bands were observed in the K-null Ub compared to the WT $\mathrm{Ub}$ pulldown (Figure 2A). The presence of multiple high molecular mass bands in the K-null Ub sample suggests that TRP32 is modified on multiple lysine residues while the presence of additional higher molecular mass bands in the WT Ub pulldown indicated TRP32 polyubiquitination. Next, we 
A

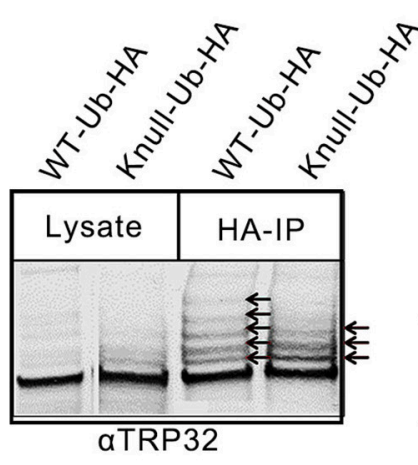

C
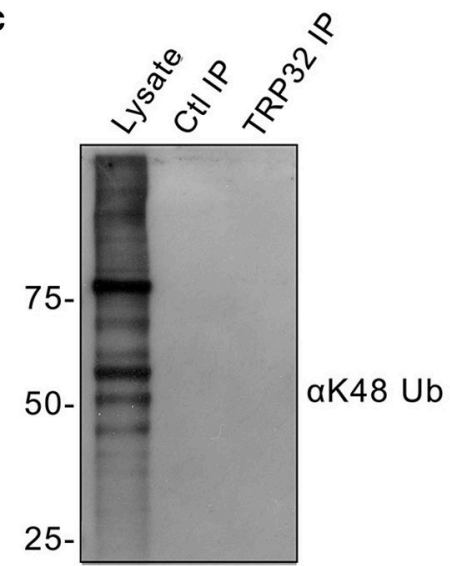

aTRP32

D

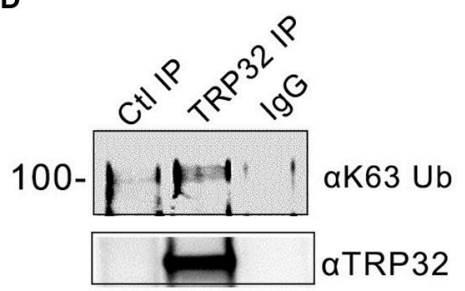

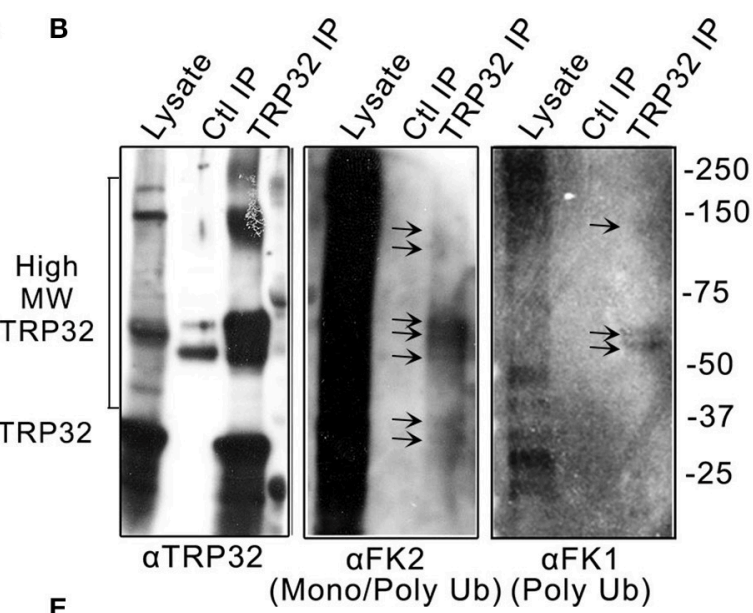

E
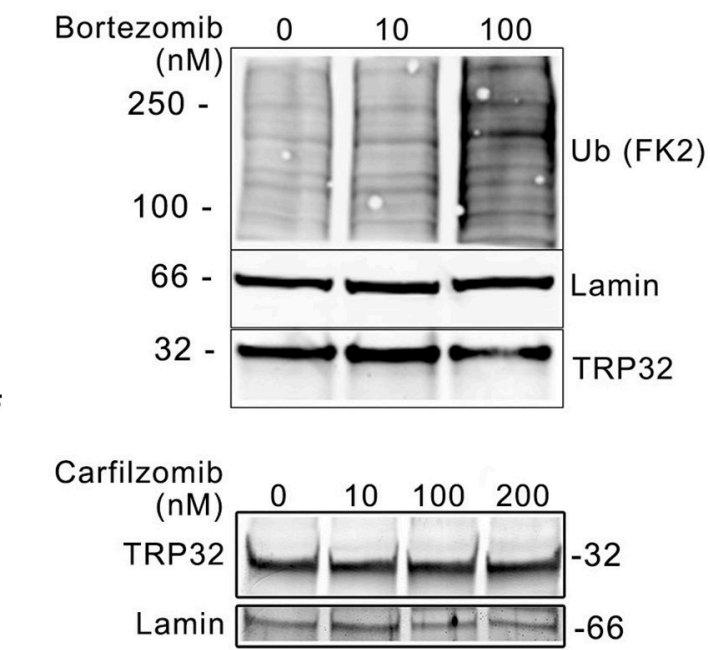

FIGURE 2 | E. chaffeensis TRP32 is mono and polyubiquitinated, but is not degraded by the proteasome. (A) GFP-tagged TRP32 was cotransfected into HeLa cells with HA-tagged WT and K-null Ub constructs. HA-immunoprecipitation was performed and the resulting eluate was probed with anti-TRP32 specific antibody by immunoblot. Multiple higher molecular weight bands indicative of ubiquitinated species of TRP32 were detected (arrows). (B) Multiple species of ubiquitinated TRP32 were also detected during infection of THP-1 cells. Anti-TRP32 specific antibody was used to immunoprecipitate TRP32 from infected THP-1 lysate. The resultant immunoprecipitated protein was probed with antibodies specific for conjugated mono and polyUb chains (FK2) and for polyUb chains alone (FK1) by immunoblot. Bands were detected (arrows) between 25 and $37 \mathrm{kDa}, 50$ and $75 \mathrm{kDa}$, and above $100 \mathrm{kDa}$ that colocalized with TRP32 bands. Immunoprecipitation of TRP32 and immunoblot with anti-K48 and anti-K63 Ub antibodies show absence of K48 ubiquitination (C) and presence of K63-linked polyubiquitinated TRP32 (D). TRP32 levels were unaffected by treatment with a proteasome inhibitor. E. chaffeensis-infected cells were incubated with varying concentrations of the proteasome inhibitor bortezomib (E) for $12 \mathrm{~h}$ or carfilzomib (F) for $8 \mathrm{~h}$ prior to harvest and immunoblotting with anti-TRP32 and anti-lamin (ctrl) and anti-Ub (FK2) antibodies.

examined TRP32 ubiquitination during infection by performing an IP using a TRP32-specific antibody or a serum control and probing the eluate with antibodies specific for polyUb (FK1) and mono- and polyUb conjugates (FK2). Ub-positive bands that coincided with detection of TRP32 (Figure 2B, panel 1) were detected at $\sim 37,40, \sim 60$, and $\sim 100 \mathrm{kDa}$ by the FK2 antibody (mono- and polyUb) (Figure 2B, panel 2); polyubiquitinated TRP32 was detected at $\sim 60$ and $100 \mathrm{kDa}$ by the FK1 antibody (polyUb specific; Figure 2B, panel 3). These findings are consistent with the presence of both multi-mono and polyubiquitinated TRP32. When the eluate was further probed using an antibody specific for K48-linked polyUb chains, none were detected (Figure 2C); however, K63Ub was detected on TRP32 (Figure 2D). Further, TRP32 did not appear 
to be degraded by the proteasome as treatment with varying concentrations of the proteasome inhibitors carfilzomib (IC50< $5 \mathrm{nM}$ ) and bortezomib (IC50 $<10 \mathrm{nM}$ ) did not alter TRP32 proteins levels (Figures 2E,F). These findings support the conclusion that the polyUb chains decorating TRP32 are not canonical K48-linked chains.

\section{TRP32 Is Ubiquitinated by NEDD4L with K63-Linked PolyUB}

We have demonstrated that NEDD4L is a HECT E3 Ub ligase that is upregulated during infection, colocalizes with ehrlichial morulae, and interacts and ubiquitinates TRP120 (Zhu et al., 2017). We examined TRP32 and identified multiple motifs that resemble the proline-rich NEDD4L interaction motif. Therefore, we examined the role of NEDD4L in TRP32 ubiquitination. To determine if TRP32 and NEDD4L interact during infection, immunoprecipitation was performed on E. chaffeensis-infected THP-1 cell lysate using either an anti-TRP32 antibody or serum control, and the resulting eluate was probed with anti-NEDD4L antibody. NEDD4L precipitated with TRP32, suggesting an interaction during infection (Figure 3A). To investigate this further, we performed an in vitro ubiquitination assay on recombinant GST-tagged TRP32 in the presence and absence of recombinant NEDD4L. We found an increased abundance of high molecular weight isoforms of TRP32 when NEDD4L was included (Figure 3B, left panel). Because NEDD4L primarily catalyzes the linkage of K63-linkaged polyUb chains, we probed the in vitro ubiquitination reactions with an anti-K63 linkagespecific antibody. We observed a strong band at $\sim 60 \mathrm{kDa}$, and a fainter band above $150 \mathrm{kDa}$ by Western immunoblot that were consistent in mass to bands detected in the TRP32 immunoblot, demonstrating that NEDD4L was able to catalyze TRP32 K63-linked polyubiquitination (Figure 3B, right panel). Additionally, when E. chaffeensis-infected cells were treated with heclin, an inhibitor of HECT family ligases including NEDD4L, some higher molecular weight isoforms of TRP32 ( $\sim 45$ and $\sim 130 \mathrm{kDa}$ ) were decreased compared to untreated cells, suggesting NEDD4L ubiquitination of TRP32 (Figure 3C). Notably, the greatest decreases were seen in the bands that appear to correspond to bands ( $\sim 65$ and $160 \mathrm{kDa})$ in GSTtagged TRP32 in the NEDD4L in vitro assay shown in Figure 3B.

\section{Ubiquitination of TRP32 Effects Subcellular Localization and Transcription Factor Function}

Because both K63-linked polyubiquitination and monoubiquitination have been linked to regulation of substrate localization, we examined the role of ubiquitination in TRP32 localization within the host cell. First, we examined the localization of polyubiquitinated TRP32. Infected cells were harvested and nuclear cytosolic fractionation performed before applying the lysates to Ub enrichment beads. The resulting eluate was probed with a TRP32-specific antibody. Higher molecular weight TRP32 was enriched in both the cytosolic $(\sim 60 \mathrm{kDa})$ and nuclear $(\sim 60$ and $150 \mathrm{kDa})$ fractions. However, different species were found in the different compartments, with $\sim 150-\mathrm{kDa}$ isoform found only in the nuclear fraction (Figure 4A). This suggested that ubiquitination may indeed play a role in TRP32 compartment-specific localization or function. To further address this, we investigated the role of $\mathrm{Ub}$ in TRP32 localization by ectopically expressing TRP32 in cells that had been treated with either an E1 inhibitor (Pyr41, pan-ubiquitination inhibitor), a HECT E3 Ub ligase-specific inhibitor (heclin), or a proteasome inhibitor (bortezomib). We found that treatment with Pyr41 resulted in gross alterations in TRP32 localization with the majority of TRP32 occurring in the cytosol and perinuclear regions. Treatment with heclin did not cause major alterations in localization, although subtle differences in subnuclear localization were seen, including increased nuclear speckles and granularity. Neither of these phenotypes were due to accumulation of undegraded cellular protein as treatment with the proteasome inhibitor bortezomib did not alter cellular localization of TRP32 (Figure 4B). In order to test this possibility, we performed a cellular assay using a firefly luciferase reporter expressed under control of the TRP32 target promoters in the presence or absence of these inhibitors (Farris et al., 2016). We found that Pyr41 caused drastic decreases in expression of both the constitutively transcribed Renilla luciferase and the firefly luciferase suggesting that this inhibitor broadly dysregulated transcription (data not shown). However, when heclin was used, we found that TRP32-mediated repression of firefly luciferase was removed and that expression returned to levels similar to the control (Figure 5).

Next, in order to confirm that transcription repression by TRP32 is due to direct ubiquitination of TRP32 and to try to identify a particular lysine residue involved, a series of lysine mutants were created, including a K-null mutant in which all four lysines were deleted. When these mutants were ectopically expressed, the K63, K93, and K123 mutants showed phenotypes characterized by peri-nucleolar rings and puncta, similar to that seen in the heclin treated cells (Figure 6). This phenotype was also observed with the K-null mutant. However, the K133 mutant showed localization similar to WT. Additionally, while the K63, K93, and K123 showed increased protein at the nuclear periphery, the K-null mutant showed much higher levels of cytosolic TRP32. When these mutants were used in a luciferase assay we found that the K-null TRP32 was unable to repress transcription of its target (Figure 7; Figure S1). Transcriptional repression was also relieved by the K63, K93, and the K123 single mutants. Although our in vitro Ub array indicated that K93 was not ubiquitinated, in silico analysis predicted K93 ubiquitination with high confidence. The cellular localization phenotype of the K93 mutant as well as transcriptional data indicates that it is also ubiquitinated. K133 which does not appear to be ubiquitinated exhibited localization similar to $\mathrm{WT}$, and was able to repress target gene transcription similar to WT TRP32.

\section{DISCUSSION}

The identification of bacterial effectors and defining their function and regulation within the host cell is essential for 


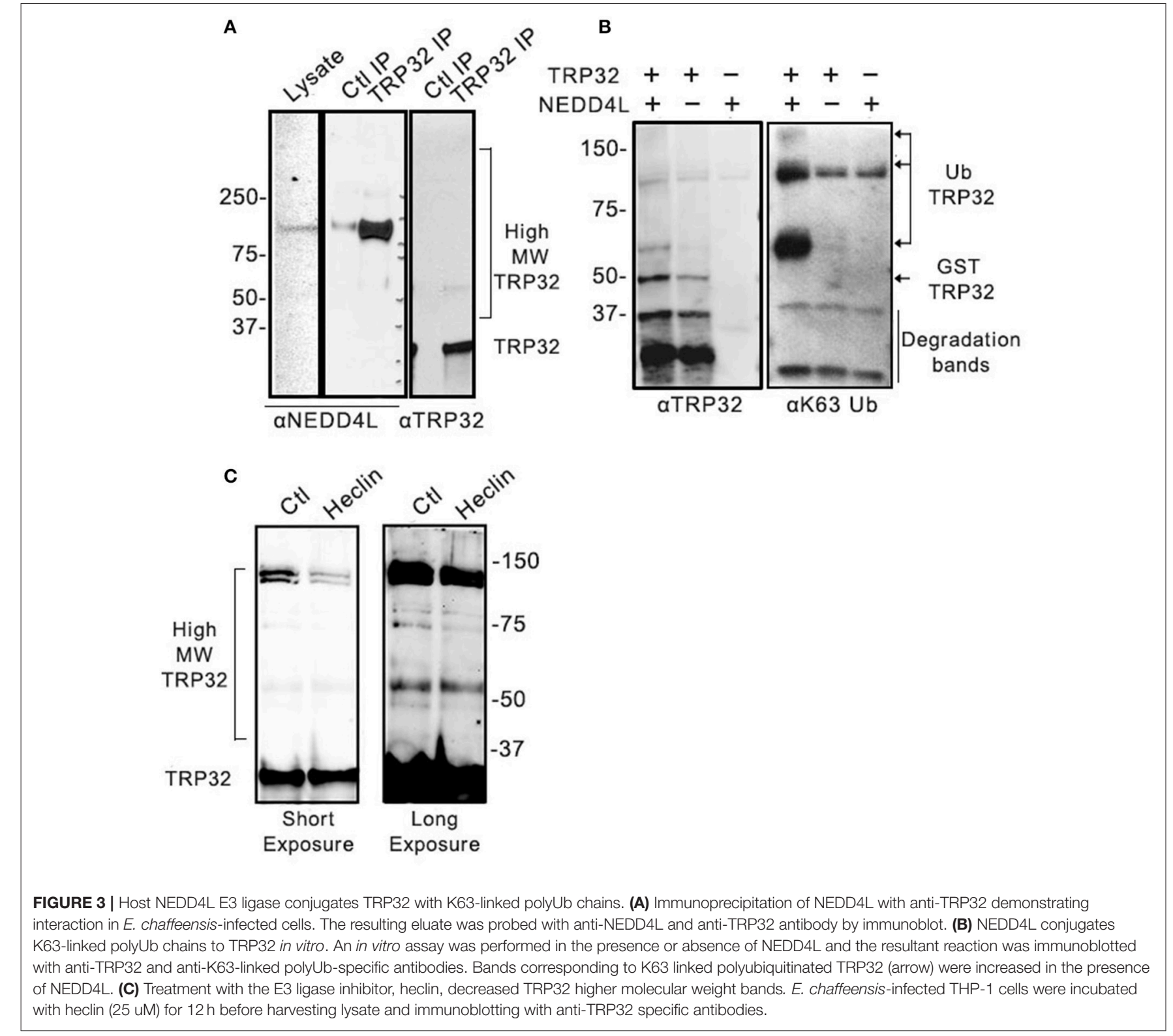

B

understanding how obligately intracellular bacteria such as E. chaffeensis manipulate the host cell for survival. Previously, we identified TRP32 as a nucleomodulin that regulated transcription of host genes related to cellular differentiation and proliferation (Farris et al., 2016). We have also recently demonstrated that NEDD4L is involved in ubiquitination of E. chaffeensis TRP120 (Zhu et al., 2017). In this study, we explored how E. chaffeensis exploits the host Ub system to regulate TRP32 localization and function.

Previously, we have shown that TRP32 is a dual function transcription factor (Farris et al., 2016). Although TRP32 possesses putative TADs and was able to activate gene transcription in yeast during infection of a mammalian cell line, the majority of TRP32 targets were downregulated and only a few were upregulated. This was also seen in a luciferase assay using TRP32 gene targets (Farris et al., 2016). Typically, dual function transcription factors that can recruit either coactivators or corepressors function in one of two ways. For some, transcription factor function is promoter-dependent, while for others, function can switch between activation and repression in a signal-dependent manner, typically by phosphorylation, SUMOylation or ubiquitination of the transcription factor (Ross et al., 2002; Boyle and Després, 2010). TRP32 is predicted to be serine/threonine phosphorylated at several sites, and tyrosine ubiquitination has been shown to play a role in TRP32 nuclear localization. Since these modifications may be required for transcription factor function, we focused on the role of $\mathrm{Ub}$, in part because TRP32 has no predicted SUMOylation sites, and SUMOylation was not detected during infection or in vitro (data not shown). Additionally, the Ub-proteasome system plays 
A

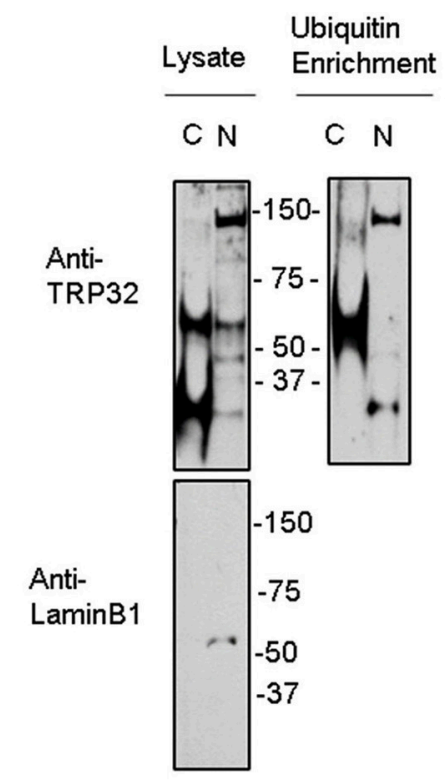

B
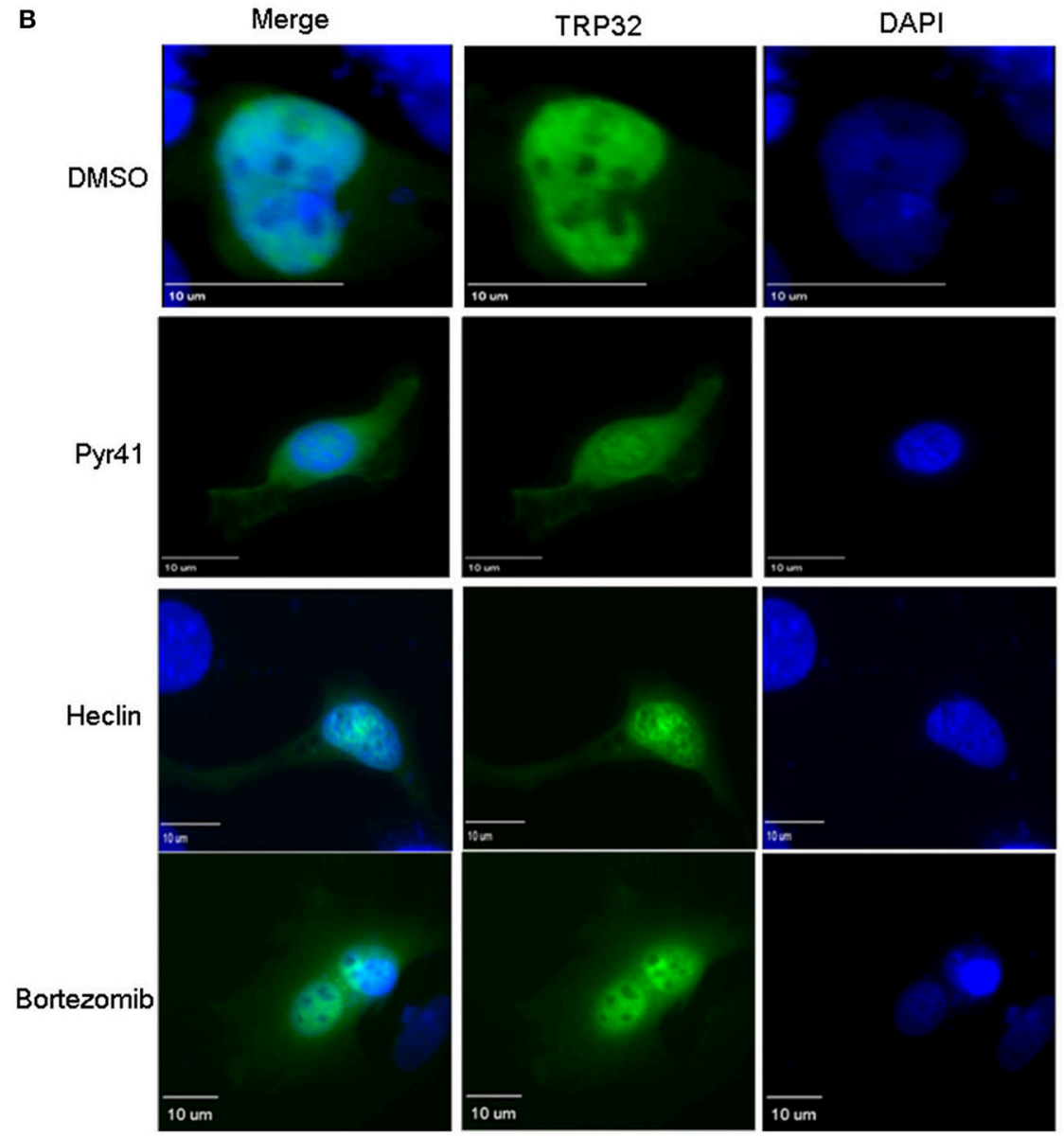

FIGURE 4 | Ubiquitination inhibitors cause altered subcellular localization of GFP-tagged TRP32. (A) Polyubiquitinated TRP32 is detected in both the nucleus and cytosol of infected cells. E. chaffeensis-infected THP-1 cells were harvested and subject to nuclear cytosolic fractionation before Ub enrichment. Lysate and eluate from Ub enrichment were probed with anti-TRP32 specific antibody by immunoblot. (B) HeLa cells were transfected with GFP-tagged TRP32. After $6 \mathrm{~h}$ the medium was changed and Pyr41 $(50 \mu \mathrm{M})$, heclin $(25 \mu \mathrm{M})$, bortezomib $(100 \mathrm{nM})$ or DMSO (vehicle) were added. At $24 \mathrm{~h}$ post-transfection cells were fixed and visualized using fluorescent microscopy. Nuclei are stained blue (DAPI). 


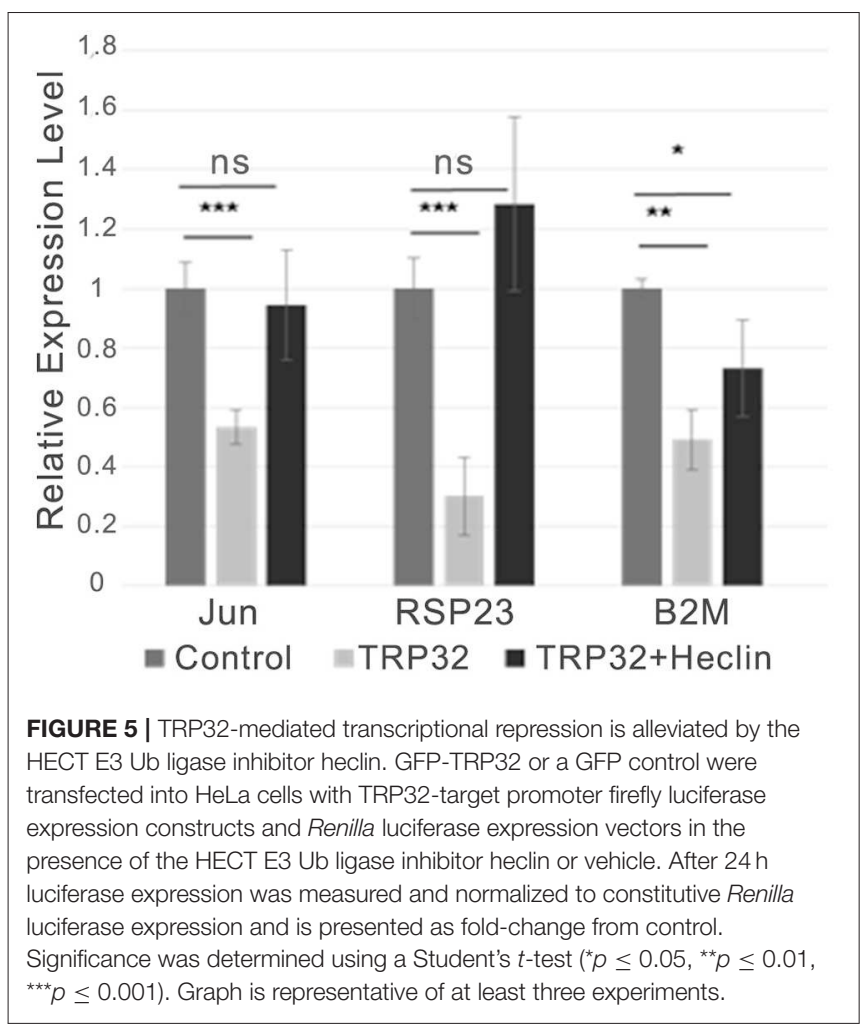

an important role in both positively and negatively regulating several transcription factors. Interestingly, several transcription factors including VP19 and Myc that possess a 9 amino acid TAD (also identified in TRP32), require polyubiquitination for TAD function (Salghetti et al., 2000). Additionally, transcription factor activation is intimately linked to proteasomal degradation in a wide variety of eukaryotic transcription factors (Molinari et al., 1999). Indeed, the proteasome is integral to transcription where it performs both proteolytic and non-proteolytic functions by playing a role in coactivator recruitment, transcription elongation, and histone modification in addition to protein degradation (Geng et al., 2012). The proximity of ubiquitinated lysines to the TRP32 9aa TADs led us to consider that such a mechanism might be at play in TRP32 transcription factor function. However, our data demonstrated that ubiquitination is required for TRP32 repressor function. This may be because TRP32 is not ubiquitinated with K48-linked polyUb chains, but instead with K63-linked chains. Indeed, the TRP32 C-terminus (which lacks lysine residues) was previously shown to interact with a member of the $20 \mathrm{~S}$ proteasome, PSMB1, which is known to play a role in transcriptional regulation (O'Hara et al., 2013; Yamauchi et al., 2013). Therefore, TRP32 may be able to recruit necessary components of the proteasome independently.

K63-linked $\mathrm{Ub}$ has been tied to the function of several transcription factors including IRF1, RORyt, and FOXO family transcription factors (Schisler et al., 2008; Tran et al., 2008; Harikumar et al., 2014; Metcalf et al., 2014; Wang et al., 2015; Zhu et al., 2015; Zeman and Cimprich, 2012). Because
K63-linked Ub chains can act as scaffolds for protein-protein interaction, these chains may be required to recruit corepressors or coactivators. There is evidence that K63-linked polyUb chains can be required for the recruitment of both histone deacetylases (HDACs) and members of the transcription initiation complex (Ramakrishna et al., 2011; Wu et al., 2016). However, it remains to be determined if this K63-linked ubiquitination is the most important modification of TRP32 in terms of transcriptional regulation. The inhibitor data with heclin appears to support this conclusion as NEDD4L is known to primarily conjugate K63-linked Ub chains to its targets. However, it also has been shown to conjugate monoUb as well as K6, K11, K26, and K29 atypical chains (Ding et al., 2013; Berndsen and Wolberger, 2014; Michel et al., 2015). Additionally, a recent study reported that one of the reasons that K63-linked chains are not linked to proteasomal degradation may be due to competitive interactions with members of ESCRT0 (Nathan et al., 2013). Although not addressed by this study, other research also implicates K63-linked chains in multivesicular body biogenesis and cargo sorting, suggesting K63-linked polyUb could play a role in TRP32 release from the ehrlichial vacuole or in intracellular trafficking.

Notably, our data suggests that ubiquitination at all three lysine residues (K63, K93, and $\mathrm{K} 123$ ) is required for TRP32 repressor function, as mutation of any one of these three lysine residues resulted in alleviation of TRP32-mediated transcriptional repression. Ubiquitination of these lysine targets were correctly predicted by the in silico analysis, and 2 of the 3 were confirmed by the in vitro peptide. Whether these lysine residues are modified by multi-monoUb, multi-polyUb, or a mix of the two remains to be determined. Each of these scenarios has been described in the literature and indeed a pattern of complex, multifunctional Ub modifications is typical of many eukaryotic transcription factors and TRP32 seems to be an adept mimic.

We found that TRP32 interacted with, and was ubiquitinated by the human E3 Ub ligase NEDD4L during infection and in vitro. We have recently shown NEDD4L interacts and ubiquitinates E. chaffeensis effector TRP120 (Zhu et al., 2017). Although TRP32 lacks the canonical NEDD4L PPxY interaction motif, it does contain three similar C-terminal motifs (TPYY, NPYY, and TPDY) that may mediate interactions with NEDD4L. Other NEDD family members have been shown to interact with (pS/pT) PxY motifs in addition to the canonical PPxY motif (Lu et al., 1999). Additionally, several known NEDD4L targets lack the PPxY motif completely and interact with NEDD4L via adaptor proteins, which may be the case with TRP32 (Mund and Pelham, 2009; de Groot et al., 2014).

Although NEDD4L has not previously been studied in the context of other bacteria, it has been linked to viral infections including HIV infection where K63-linked ubiquitination of the viral protein Gag by NEDD4L plays an important role in viral budding and in enterovirus 71 where it may function via altering IFN- $\beta$ production (Chung et al., 2008; Kuo et al., 2015). In uninfected human cells, NEDD4L has been most commonly studied in the context of the regulation of membrane proteins such as sodium and potassium channels which are 


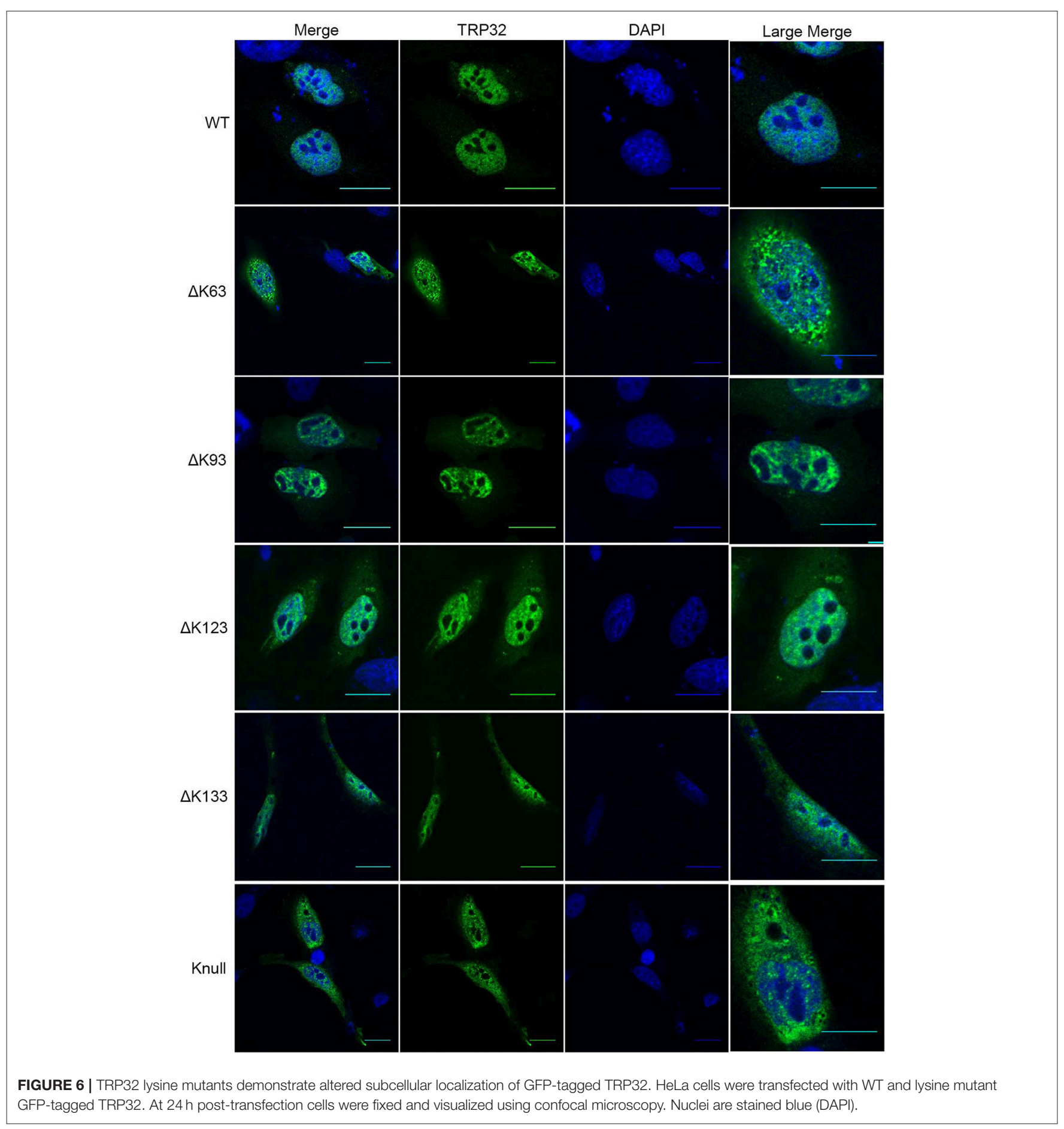

directed to recycling endosomes by NEDD4L (Scheffner and Kumar, 2014). However, NEDD4L has also been shown to be an important regulator of the TGF- $\beta$ and Wnt signaling pathway via ubiquitination of SMADs and Disheveled proteins, respectively (Gao et al., 2009; Ding et al., 2013). Especially interesting in the context of Ehrlichia infection is that NEDD4L is phosphorylated and activated by JNK in a WNT5a-dependent manner (Ding et al., 2013). Previously, we have shown that both canonical and non-canonical Wnt signaling are vitally important for successful E. chaffeensis infection, and WNT5a knockdown results in significantly decreased ehrlichial infection (Luo et al., 2015). It is likely that WNT5a activation of NEDD4L plays a role in licensing TRP32 transcription factor function. It may do this by regulating TRP32s interactions with host proteins as was demonstrated with TRP120 (Zhu et al., 2017). 


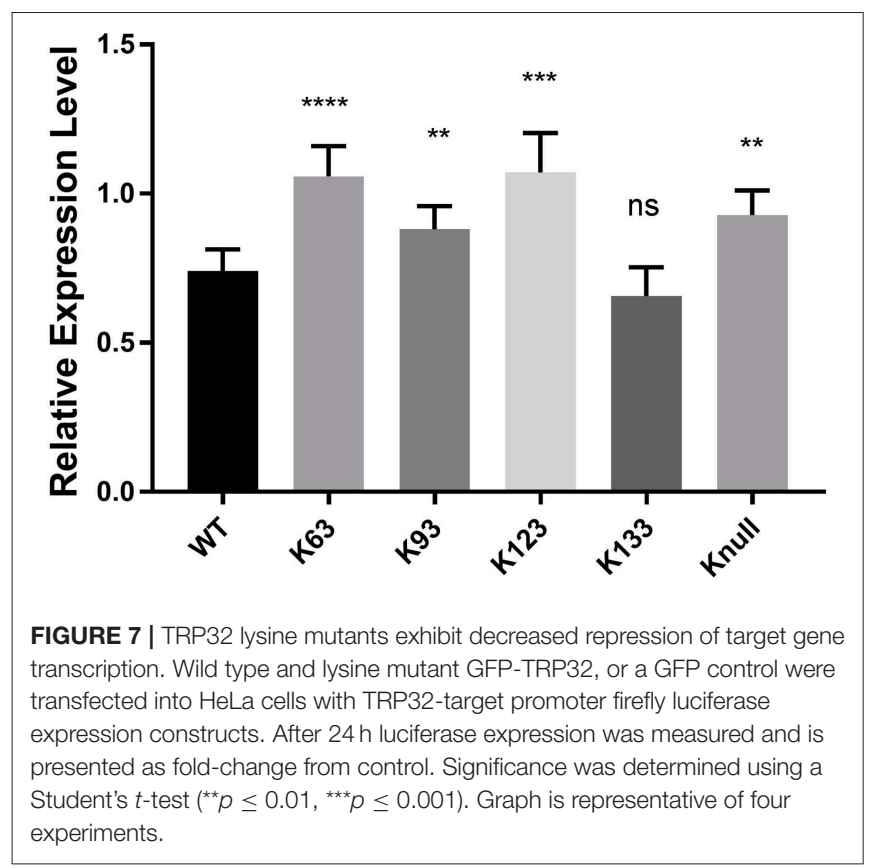

During infection, we determined that TRP32 interacts with NEDD4L and can be ubiquitinated by NEDD4L in vitro. Multiple mono- and polyubiquitinated forms of TRP32 were detected during infection, including some which did not completely disappear when cells were treated with the NEDD4L inhibitor. Further, we found that treatment with heclin both impaired the ability of TRP32 to repress target gene transcription and altered its subnuclear localization, and that mutation of specific TRP32 lysine residues mimicked these phenotypes. It is likely that ubiquitination may serve multiple functions in regulating TRP32 and that other Ub ligases are required in addition to

\section{REFERENCES}

Anderson, D. M., and Frank, D. W. (2012). Five mechanisms of manipulation by bacterial effectors: a ubiquitous theme. PLoS Pathog. 8:e1002823. doi: 10.1371/journal.ppat.1002823

Ashida, H., Kim, M., and Sasakawa, C. (2014). Exploitation of the host ubiquitin system by human bacterial pathogens. Nat. Rev. Micro. 12, 399-413. doi: $10.1038 /$ nrmicro3259

Berndsen, C. E., and Wolberger, C. (2014). New insights into ubiquitin E3 ligase mechanism. Nat. Struct. Mol. Biol. 21, 301-307. doi: 10.1038/nsmb. 2780

Boyle, P., and Després, C. (2010). Dual-function transcription factors and their entourage: unique and unifying themes governing two pathogenesisrelated genes. Plant Signal. Behav. 5, 629-634. doi: 10.4161/psb.5. 6.11570

Chung, H.-Y., Morita, E., von Schwedler, U., Müller, B., Kräusslich, H.-G., and Sundquist, W. I. (2008). NEDD4L overexpression rescues the release and infectivity of human immunodeficiency virus Type 1 constructs lacking PTAP and YPXL late domains. J. Virol. 82, 4884-4897. doi: 10.1128/JVI.0 2667-07

Collins, C. A., and Brown, E. J. (2010). Cytosol as battleground: ubiquitin as a weapon for both host and pathogen. Trends Cell Biol. 20, 205-213. doi: 10.1016/j.tcb.2010.01.002
NEDD4L. Indeed, TRP32 is known to react with the putative RING-type E3 Ub ligase Roquin (Luo and McBride, 2012). Future directions include identifying the mechanism of altered TRP32 transcriptional function mediated by ubiquitination, fully characterizing the $\mathrm{Ub}$ modifications that occur on the various TRP32 lysine residues, and examining the potential crosstalk between these various Ub modifications and other TRP32 PTMs.

\section{AUTHOR CONTRIBUTIONS}

TF: Plan research, perform experiments, manuscript prep, and editing. BZ: Plan research, perform experiments. JW: Manuscript prep and editing. JM: Plan research, manuscript prep and editing.

\section{FUNDING}

This work was supported by the National Institutes of Allergy and Infectious Diseases grants AI105536 and AI106859 (to JM). Tierra Farris was supported by the NIAID T32 AI 007526-15 and by a James W. McLaughlin Predoctoral Fellowship.

\section{ACKNOWLEDGMENTS}

We are grateful to all of the lab members both current and past for their insight, advice, and many useful discussions.

\section{SUPPLEMENTARY MATERIAL}

The Supplementary Material for this article can be found online at: https://www.frontiersin.org/articles/10.3389/fcimb. 2017.00534/full\#supplementary-material

Figure S1 | Expression levels of TRP32 wild type and lysine mutant expression constructs in HeLa cells.

de Groot, T., Trimpert, C., Wesche, D., Wong, V., van den Berg, D., Stagljar, I., et al. (2014). NDFIP1: the missing adaptor for aquaporin-2 regulation by NEDD4 and NEDD4L (LB723). FASEB J. 28(Suppl. 1):LB723. doi: 10.1096/fj.153 $0-6860$

Ding, Y., Zhang, Y., Xu, C., Tao, Q. H., and Chen, Y. G. (2013). HECT Domaincontaining E3 Ubiquitin Ligase NEDD4L negatively regulates Wnt signaling by targeting dishevelled for proteasomal degradation. J. Biol. Chem. 288, 8289-8298. doi: 10.1074/jbc.M112.433185

Dunphy, P. S., Luo, T., and McBride, J. W. (2014). Ehrlichia chaffeensis exploits host SUMOylation pathways to mediate effector-host interactions and promote intracellular survival. Infect. Immun. 82, 4154-4168. doi: 10.1128/IAI.0 1984-14

Farris, T. R., Dunphy, P. S., Zhu, B., Kibler, C. E., and McBride, J. W. (2016). Ehrlichia chaffeensis TRP32 is a nucleomodulin that directly regulates expression of host genes governing differentiation and prolimferation. Infect. Immun. 84, 3184-3194. doi: 10.1128/IAI.00657-16

Gao, S., Alarcón, C., Sapkota, G., Rahman, S., Chen, P.-Y., Goerner, N., et al. (2009). Ubiquitin Ligase Nedd4L targets activated Smad2/3 to limit TGF- $\beta$ signaling. Mol. Cell 36, 457-468. doi: 10.1016/j.molcel.2009. 09.043

Geng, F., Wenzel, S., and Tansey, W. P. (2012). Ubiquitin and Proteasomes in transcription. Annu. Rev. Biochem. 81, 177-201. doi: 10.1146/annurev-bioche $\mathrm{m}-052110-120012$ 
Harikumar, K. B., Yester, J. W., Surace, M. J., Oyeniran, C., Price, M. M., Huang, W.-C., et al. (2014). K63-linked polyubiquitylation of IRF1 transcription factor is essential for IL-1-induced CCL5 and CXCL10 chemokine production. Nat. Immunol. 15, 231-238. doi: 10.1038/ni.2810

Komander, D. (2009). The emerging complexity of protein ubiquitination. Biochem. Soc. Trans. 37(Pt 5), 937-953. doi: 10.1042/BST03 70937

Kuo, R.-L., Lin, Y.-H., Wang, R. Y.-L., Hsu, C.-W., Chiu, Y.-T., Huang, H.-I., et al. (2015). Proteomics analysis of EV71-infected cells reveals the involvement of host protein NEDD4L in EV71 replication. J. Proteome Res. 14, 1818-1830. doi: $10.1021 /$ pr501199h

Kuriakose, J. A., Zhang, X., Luo, T., and McBride, J. W. (2012). Molecular basis of antibody mediated immunity against Ehrlichia chaffeensis involves species-specific linear epitopes in tandem repeat proteins. Microbes Infect. 14, 1054-1063. doi: 10.1016/j.micinf.2012.05.012

Lina, T. T., Dunphy, P. S., Luo, T., and McBride, J. W. (2016a). Ehrlichia chaffeensis TRP120 Activates canonical Notch signaling to downregulate TLR2/4 expression and promote intracellular survival. MBio 7:e00672-16. doi: $10.1128 / \mathrm{mBio} .00672-16$

Lina, T. T., Farris, T., Luo, T., Mitra, S., Zhu, B., and McBride, J. W. (2016b). Hacker within! Ehrlichia chaffeensis effector driven phagocyte reprogramming strategy. Front. Cell. Infect. Microbiol. 6:58. doi: 10.3389/fcimb.2016. 00058

Lu, P.-J., Zhou, X. Z., Shen, M., and Lu, K. P. (1999). Function of WW Domains as phosphoserine- or phosphothreonine-binding modules. Science 283, 13251328. doi: $10.1126 /$ science.283.5406.1325

Luo, T., Dunphy, P. S., Lina, T. T., and McBride, J. W. (2015). Ehrlichia chaffeensis exploits canonical and noncanonical host Wnt signaling pathways to stimulate phagocytosis and promote intracellular survival. Infect. Immun. 84, 686-700. doi: 10.1128/IAI.01289-15

Luo, T., and McBride, J. W. (2012). Ehrlichia chaffeensis TRP32 interacts with host cell targets that influence intracellular survival. Infect. Immun. 80, 2297-2306. doi: 10.1128/IAI.00154-12

Luo, T., Zhang, X., Wakeel, A., Popov, V. L., and McBride, J. W. (2008). A variable-length PCR target protein of Ehrlichia chaffeensis contains major species-specific antibody epitopes in acidic serinerich tandem repeats. Infect. Immun. 76, 1572-1580. doi: 10.1128/IAI.0 1466-07

McBride, J. W., Zhang, X., Wakeel, A., and Kuriakose, J. A. (2011). Tyrosinephosphorylated Ehrlichia chaffeensis and Ehrlichia canis tandem repeat orthologs contain a major continuous cross-reactive antibody epitope in lysine-rich repeats. Infect. Immun. 79, 3178-3187. doi: 10.1128/IAI.0 1347-10

McLean, C. Y., Bristor, D., Hiller, M., Clarke, S. L., Schaar, B. T., Lowe, C. B., et al. (2010). GREAT improves functional interpretation of cis-regulatory regions. Nat. Biotechnol. 28, 495-501. doi: 10.1038/nbt.1630

Metcalf, J. L., Bradshaw, P. S., Komosa, M., Greer, S. N., Stephen Meyn, M., and Ohh, M. (2014). K63-Ubiquitylation of VHL by SOCS1 mediates DNA double-strand break repair. Oncogene 33, 1055-1065. doi: 10.1038/onc. 2013.22

Metzger, M. B., Hristova, V. A., and Weissman, A. M. (2012). HECT and RING finger families of E3 ubiquitin ligases at a glance. J. Cell Sci. 125, 531-537. doi: $10.1242 /$ jcs.091777

Michel, M. A., Elliott, P. R., Swatek, K. N., Simicek, M., Pruneda, J. N., Wagstaff, J. L., et al. (2015). Assembly and specific recognition of k29- and k33-linked polyubiquitin. Mol. Cell 58, 95-109. doi: 10.1016/j.molcel.2015. 01.042

Molinari, E., Gilman, M., and Natesan, S. (1999). Proteasome-mediated degradation of transcriptional activators correlates with activation domain potency in vivo EMBO J. 18, 6439-6447.

Mund, T., and Pelham, H. R. B. (2009). Control of the activity of WW-HECT domain E3 ubiquitin ligases by NDFIP proteins. EMBO Rep. 10, 501-507. doi: 10.1038 /embor.2009.30

Nathan, J. A., Kim, H. T., Ting, L., Gygi, S. P., and Goldberg, A. L. (2013). Why do cell proteins linked to K63-polyubiquitin chains not associate with proteasomes? EMBO J. 32, 552-565. doi: 10.1038/emboj.2012.354

O'Hara, A., Howarth, A., Varro, A., and Dimaline, R. (2013). The role of proteasome beta subunits in gastrin-mediated transcription of plasminogen activator inhibitor-2 and regenerating protein1. PLOS ONE 8:e59913. doi: 10.1371/journal.pone.0059913

Patel, J. C., Hueffer, K., Lam, T. T., and Galán, J. E. (2009). Diversification of a Salmonella virulence protein function by ubiquitin-dependent differential localization. Cell 137, 283-294. doi: 10.1016/j.cell.2009. 01.056

Popa, C. M., Tabuchi, M., and Valls, M. (2016). Modification of bacterial effector proteins inside Eukaryotic host cells. Front. Cell. Infect. Microbiol. 6:73. doi: 10.3389/fcimb.2016.00073

Radivojac, P., Vacic, V., Haynes, C., Cocklin, R. R., Mohan, A., Heyen, J. W., et al. (2010). Identification, analysis, and prediction of protein ubiquitination sites. Proteins 78, 365-380. doi: 10.1002/prot.22555

Ramakrishna, S., Suresh, B., Lee, E.-J., Lee, H.-J., Ahn, W.-S., and Baek, K.H. (2011). Lys-63-specific Deubiquitination of SDS3 by USP17 Regulates HDAC activity. J. Biol. Chem. 286, 10505-10514. doi: 10.1074/jbc.M110. 162321

Ravikumar, V., Jers, C., and Mijakovic, I. (2015). Elucidating host-pathogen interactions based on post-translational modifications using proteomics approaches. Front. Microbiol. 6:1313. doi: 10.3389/fmicb.2015.01312

Ribet, D., and Cossart, P. (2010). Post-translational modifications in host cells during bacterial infection. FEBS Lett. 584, 2748-2758. doi: 10.1016/j.febslet.2010.05.012

Ross, S., Best, J. L., Zon, L. I., and Gill, G., (2002). SUMO-1 Modification Represses Sp3 transcriptional activation and modulates its subnuclear localization. Mol. Cell 10, 831-842. doi: 10.1016/S1097-2765(02)00682-2

Salghetti, S. E., Muratani, M., Wijnen, H., Futcher, B., and Tansey, W. P. (2000). Functional overlap of sequences that activate transcription and signal ubiquitin-mediated proteolysis. Proc. Natl. Acad. Sci. U.S.A. 97, 3118-3123. doi: $10.1073 /$ pnas.97.7.3118

Salmena, L., and Pandolfi, P. P. (2007). Changing venues for tumour suppression: balancing destruction and localization by monoubiquitylation. Nat. Rev. Cancer 7, 409-413. doi: 10.1038/nrc2145

Scheffner, M., and Kumar, S. (2014). Mammalian HECT ubiquitin-protein ligases: biological and pathophysiological aspects. Biochim. Biophys. Acta 1843, 61-74. doi: 10.1016/j.bbamcr.2013.03.024

Schindelin, J., Arganda-Carreras, I., Frise, E., Kaynig, V., Longair, M., Pietzsch, T., et al. (2012). Fiji: an open-source platform for biological-image analysis. Nat. Methods 9, 676-682. doi: 10.1038/nmeth.2019

Schisler, J. C., Willis, M. S., and Patterson, C. (2008). You spin me round: mafBx/Atrogin-1 feeds forward on FOXO transcription factors (like a record). Cell Cycle 7, 440-443. doi: 10.4161/cc.7.4.5451

Shirai, A., Matsuyama, A., Yashiroda, Y., Hashimoto, A., Kawamura, Y., Arai, R., et al. (2008). Global analysis of gel mobility of proteins and its use in target identification. J. Biol. Chem. 283, 10745-10752. doi: 10.1074/jbc.M709211200

Thomas, M., and Holden, D. W. (2009). Ubiquitination - a bacterial effector's ticket to ride. Cell Host Microbe 5, 309-311. doi: 10.1016/j.chom.2009. 03.010

Tran, H., Hamada, F., Schwarz-Romond, T., and Bienz, M. (2008). Trabid, a new positive regulator of Wnt-induced transcription with preference for binding and cleaving K63-linked ubiquitin chains. Genes Dev. 22, 528-542. doi: $10.1101 /$ gad.463208

Wakeel, A., den Dulk-Ras, A., Hooykaas, P. J., and McBride, J. W. (2011). Ehrlichia chaffeensis tandem repeat proteins and Ank200 are type 1 secretion system substrates related to the repeats-in-toxin exoprotein family. Front. Cell. Infect. Microbiol. 1:22. doi: 10.3389/fcimb.2011.00022

Wang, X., Yang, J., Han, L., Zhao, K., Wu, Q., Bao, L., et al. (2015). TRAF5-mediated K63-linked polyubiquitination play essential role in positive regulation of ROR $\gamma \mathrm{t}$ on promoting IL-17A expression. J. Biol. Chem. 290, 29086-29094. doi: 10.1074/jbc.M115.664573

Wu, H.-T., Kuo, Y.-C., Hung, J.-J., Huang, C.-H., Chen, W.-Y., Chou, T.-Y., et al. (2016). K63-polyubiquitinated HAUSP deubiquitinates HIF- $1 \alpha$ and dictates H3K56 acetylation promoting hypoxia-induced tumour progression. Nat. Commun. 7:13644. doi: 10.1038/ncomms 13644

Yamauchi, J., Sekiguchi, M., Shirai, T., Yamada, M., and Ishimi, Y. (2013). Role of nuclear localization of PSMB1 in transcriptional activation. Biosci. Biotechnol. Biochem. 77, 1785-1787. doi: 10.1271/bbb. 130290 
Zeman, M. K., and Cimprich, K. A. (2012). Finally, Polyubiquitinated PCNA gets recognized. Mol. Cell 47, 333-334. doi: 10.1016/j.molcel.2012. 07.024

Zhu, B., Das, S., Mitra, S., Farris, T. R., and McBride, J. W. (2017). Ehrlichia chaffeensis TRP120 moonlights as a HECT E3 ligase involved in self and host ubiquitination to influence protein interactions and stability for intracellular survival. Infect. Immun. 85:e00290-17. doi: 10.1128/IAI.00290-17

Zhu, B., Farris, T., Milligan, S., Chen, H. S., Zhu, R. J., Hong, A., et al. (2016). Rapid identification of ubiquitination and SUMOylation target sites by microfluidic peptide array. Biochem. Biophys. Rep. 5, 430-438. doi: 10.1016/j.bbrep.2016.02.003

Zhu, B., Kuriakose, J. A., Luo, T., Ballesteros, E., Gupta, S., Fofanov, Y., et al. (2011). Ehrlichia chaffeensis TRP120 binds a G+C-rich motif in host cell DNA and exhibits eukaryotic transcriptional activator function. Infect. Immun. 79, 4370-4381. doi: 10.1128/IAI.05422-11
Zhu, B., Yan, K., Li, L., Lin, M., Zhang, S., He, Q., et al. (2015). K63-linked ubiquitination of FANCG is required for its association with the Rap80-BRCA1 complex to modulate homologous recombination repair of DNA interstand crosslinks. Oncogene 34, 2867-2878. doi: 10.1038/onc.2014.229

Conflict of Interest Statement: The authors declare that the research was conducted in the absence of any commercial or financial relationships that could be construed as a potential conflict of interest.

Copyright $\odot 2018$ Farris, Zhu, Wang and McBride. This is an open-access article distributed under the terms of the Creative Commons Attribution License (CC BY).

The use, distribution or reproduction in other forums is permitted, provided the original author(s) or licensor are credited and that the original publication in this journal is cited, in accordance with accepted academic practice. No use, distribution or reproduction is permitted which does not comply with these terms. 